\title{
Interaction Between Ethylene and Abscisic Acid and Maturation in Highbush Blueberry
}

\author{
Manabu Watanabe ${ }^{1 *}$, Ryota Goto ${ }^{2}$, Masanobu Murakami ${ }^{1}$, Sadao Komori $^{2}$ \\ and Akira Suzuki²
}

${ }^{1}$ Field Science Center, Faculty of Agriculture, Iwate University, Takizawa 020-0611, Japan

${ }^{2}$ Faculty of Agriculture, Iwate University, Ueda, Morioka 020-8550, Japan

The aim of the present study was to clarify the interaction between ethylene and abscisic acid (ABA) and maturation in highbush blueberry. We investigated the berry quality, ethylene evolution, and ABA concentration of berries treated with ethephon, an ethylene-generating compound; aminoethoxyvinylglycine (AVG), an ethylene synthesis inhibitor; ABA; and nordihydroguaiaretic acid (NDGA), an ABA synthesis inhibitor, before the onset of maturation in the highbush blueberry 'Jersey'. The harvest time and the increasing time of ethylene evolution and $\mathrm{ABA}$ concentration in berries were earlier in $200 \mathrm{mg} \cdot \mathrm{L}^{-1}$ ethephon treatment than in the control. Treatment of $30 \mathrm{mg} \cdot \mathrm{L}^{-1} \mathrm{ABA}$ progressed the time of increased ABA concentration but had little effect on the harvest time or ethylene evolution. AVG treatment at $150 \mathrm{mg} \cdot \mathrm{L}^{-1}$ delayed the harvest time accompanied by decreasing the peak value of ethylene evolution and delaying the time of increased ABA concentration. Treatment of $300 \mathrm{mg} \cdot \mathrm{L}^{-1}$ NDGA decreased the peak value of the ABA concentration, while it did not affect the harvesting time or ethylene evolution. These results suggest that ethylene, not $\mathrm{ABA}$, induced maturation of blueberry at the mature green stage, and that ethylene was more closely involved than $\mathrm{ABA}$ with the progress of the whole maturation process.

Key Words: aminoethoxyvinylglycine, climacteric, ethephon, nordihydroguaiaretic acid, Vaccinium corymbosum L.

\section{Introduction}

Harvesting in blueberry culture requires a great deal of labor, because berries do not mature simultaneously even in a single cluster, shoot, or tree, and berries are picked by hand, one by one. If cluster harvesting could be done in blueberry culture as in grape, it would likely save a lot of labor. There are a few reports of cluster harvesting systems in blueberry. Kobayashi et al. (2011) evaluated the suitability of four highbush blueberry cultivars for cluster harvesting. Miyashita et al. (2019) investigated whether Vaccinium corymbosum and Vaccinium virgatum hybrids were appropriate for cluster harvesting. New blueberry cultivars for cluster har-

Received; May 10, 2020. Accepted; September 16, 2020.

First Published Online in J-STAGE on November 27, 2020.

This study was supported by a Grant-in-Aid for Scientific Research (KAKENHI No. 21780019) from the Japan Society for the Promotion of Science (JSPS).

Part of this paper was presented at the Meeting of the Japanese Society for Horticultural Science held in Spring 2012.

* Corresponding author (E-mail: mwata@iwate-u.ac.jp). vesting will be bred in the near future. It is possible that a cluster harvesting system may also be used for many existing cultivars with excellent fruit qualities if plant growth regulators could control the maturation of berries. Thus, it is necessary to elucidate the roles of plant growth regulators for maturation in blueberry.

Blueberry exhibits a double-sigmoid growth pattern. The berry weight, anthocyanin concentration in the pericarp, and sugar content increase rapidly, and the tittered acid content and berry firmness decrease rapidly during stage III of the double-sigmoid curve. The respiration rate and ethylene evolution of the berry peaks after the onset of coloration during maturation in many cultivars of highbush blueberry (Shimura et al., 1986; Suzuki et al., 1997a; Windus et al., 1976). The abscisic acid (ABA) concentration of the berries increases with the coloration of the pericarp and increase in sugar content (Kobashi et al., 2002; Zifkin et al., 2012). Furthermore, Chung et al. (2019) indicated that ABA biosynthesis and signal transduction and anthocyanin biosynthesis are closely associated with anthocyanin accumulation and skin coloration in highbush blueberry 
fruit during ripening by transcriptome analysis using RNA-Seq. Ethylene evolution increased simultaneously with the ABA concentration in 'Earliblue' and 'Berkeley', and increased earlier than the ABA concentration in 'Jersey' blueberry (Goto et al., 2013). Furthermore, these results suggest that both ethylene and ABA may be related to the maturation progress in highbush blueberry.

There is controversy over whether blueberry is classified as a climacteric type or non-climacteric type. Many researchers indicated that the respiration rate and ethylene evolution were increased during the maturation period in blueberry (Goto et al., 2013; Ismail and Kender, 1969; Lipe, 1978; Shimura et al., 1986; Suzuki et al., 1997a; Windus et al., 1976). These findings support that blueberry is classified as a climacteric type. While, some reports classified blueberry as a nonclimacteric type (Chung et al., 2019; Oh et al., 2018; Zifkin et al., 2012) because the peak of the respiration rate and ethylene evolution were not observed (Frenkel, 1972; Hall and Forsyth, 1967) and there was the increase in the $\mathrm{ABA}$ concentration, such as in grape (Coombe and Hale, 1973), during the maturation period in blueberry. However, the peak of ethylene evolution could not be detected depending on the collecting interval (Ismail and Kender, 1969), since maturation progresses rapidly in blueberry (Windus et al., 1976). The ABA concentration increases during the maturation period even in climacteric types such as apple, peach, and mango (Kobashi et al., 2001; Kondo et al., 1991; Zaharah et al., 2012). Therefore, blueberry should not be classified as a non-climacteric type according to only the change in the ABA concentration and inability to determine the peak of ethylene evolution.

Generally, a burst of ethylene production acts as an initiator of ripening in climacteric fruit (Hiwasa-Tanase and Ezura, 2014). Recent studies reported that ethylene evolution was induced by $\mathrm{ABA}$ during maturation in some climacteric fruit (Mou et al., 2016; Zhang et al., 2009a). However, it is unclear which triggers maturation in blueberry, ethylene or ABA. Suzuki et al. (1997b) reported that blueberries treated with aminooxyacetic acid, the inhibitor of ethylene biosynthesis, 10 days before the onset of maturation did show inhibition of ethylene evolution, but that maturation was delayed. Oh et al. (2018) found that the application of ABA during berry development in the 'Jersey' blueberry temporarily increased the ABA level and stimulated berry coloration. However, there are no reports regarding the effect of ethylene treatment on the ABA concentration, or of ABA treatment on ethylene evolution for maturation in blueberry.

In previous reports, blueberry clusters or bushes with berries at different developmental stages were treated with plant growth regulators related to ethylene, such as ethephon and $\mathrm{ABA}$, and the effects on maturation were estimated (Ban et al., 2007; Buran et al., 2012; Eck,
1970; Oh et al., 2018). The effects of the plant growth regulators differed depending on the fruit developmental stage (Bonghi et al., 2011; Lara and Vendrell, 2000b). Therefore, it seems that the effects of plant growth regulators on fruit maturation have not yet been fully clarified.

There are many reports about the relationship between fruit maturation and plant growth regulators. Many researchers have applied plant growth regulators to fruits detached from the tree, and then investigated fruit quality, endogenous plant hormone levels, and related genes (Lara and Vendrell, 2000b; Mou et al., 2016; Zhang et al., 2009a, b). Ethylene production of attached apples, however, was delayed and lower in magnitude than values measured in detached 'Gala' apple fruits (Lin and Walsh, 2008). The possibility that wounding stress caused by harvest coupled with nutrient and water deficiencies inducing additional system 1 ethylene cannot be eliminated (Rogiers and Knowles, 1999; Walsh, 1977). Therefore, fruits attached on the tree should be utilized for the physiological study of fruit maturing on the tree.

The aim of the present study was to clarify the interaction between ethylene and ABA and maturation in highbush blueberry. We investigated the berry quality, ethylene evolution, and $\mathrm{ABA}$ concentration of berries treated with ethylene-generating compound, ABA, ethylene synthesis inhibitor, and ABA synthesis inhibitor before the onset of maturation on the tree in the highbush blueberry 'Jersey'.

\section{Materials and Methods}

Experiment 1: Effects of ethephon and ABA treatment on berries before the onset of maturation on the maturation, ethylene evolution, and $A B A$ concentration

Four 26-year-old northern highbush blueberry 'Jersey' bushes growing at Takizawa Farm at the Field Science Center of Iwate University, Japan, were used in 2010. Three canes were selected per bush, and one cane per bush was used for each treatment. Approximately 200 berries, which were light green in the pericarp at the mature green (MG) stage (Suzuki et al., 1997a), were selected per treatment and tagged just before treatment. The berries were sprayed with ethephon (Ethrel 10; Nissan Chemical Corp., Tokyo, Japan) $200 \mathrm{mg} \cdot \mathrm{L}^{-1}$ and (+)-cis, trans-abscisic acid (OlChemIm Ltd., Czech) (ABA) $30 \mathrm{mg} \cdot \mathrm{L}^{-1}$ containing $0.1 \%$ Tween 20 on 14 July. These sprays were conducted gently not to drift toward the leaves. Untreated berries were used as the control. The berries were collected randomly on $0,3,7$, 11, and 16 days after treatment (DAT) to determine the weight, quality, ethylene evolution, and ABA concentration. The berries for ABA measurement were weighed and then stored at $-20^{\circ} \mathrm{C}$ until analysis.

One to three berries were collected per treatment on each sampling day for analysis of the berry quality. Berry firmness was measured with a fruit hardness 
tester (KM-1; Fujiwara Scientific Co., Ltd, Tokyo, Japan). The juices were extracted from the 1-3 berries used for the firmness measurement, and we measured the soluble solid content using a refractometer (APAL-1; ASONE Corporation, Osaka, Japan) and the $\mathrm{pH}$ with a $\mathrm{pH}$ meter (B-212; Horiba Ltd., Kyoto, Japan). Two to four replicates were performed.

Ethylene evolution was measured following the procedure described by Suzuki et al. (1997a). The 3-4 berries collected per treatment on each sampling day were placed in a $91-\mathrm{mL}$ bottle capped with a silicon stopper and sealed with parafilm for $6 \mathrm{~h}$ at room temperature. A 1-mL gas sample was taken from the bottle with a gas tight glass syringe and analyzed by gas chromatography equipped with FID (GC-14A; Shimadzu Corporation, Kyoto, Japan). Four replicates were performed.

The extraction and purification procedures for $\mathrm{ABA}$ were performed according to Goto et al. (2013). One berry collected per treatment on each sampling day was macerated in cold $80 \%(\mathrm{v} / \mathrm{v})$ methanol containing $100 \mathrm{mg} \cdot \mathrm{L}^{-1}$ butyl hydroxytoluene (Wako Pure Chemical Industries, Ltd., Osaka, Japan) and $500 \mathrm{ng}$ $\left[{ }^{2} \mathrm{H}_{6}\right]$ ABA (OlChemIm.) as an internal standard. The homogenate was extracted overnight at $4^{\circ} \mathrm{C}$ in darkness. The extract was centrifuged $(10,000 \times \mathrm{g}, 10 \mathrm{~min}$, $4^{\circ} \mathrm{C}$ ) and then filtered, the filtrate was evaporated in vacuo at $40^{\circ} \mathrm{C}$, and the residue dissolved in phosphate buffer $(\mathrm{pH} \mathrm{8.5)}$. This solution was then centrifuged $\left(14,000 \times \mathrm{g}, 10 \mathrm{~min}, 4^{\circ} \mathrm{C}\right)$. Insoluble polyvinylpolypyrrolidone was added to the supernatant, and the solution was shaken for $20 \mathrm{~min}$, and then filtered. The filtrate was adjusted to $\mathrm{pH} 2.0$ with formic acid.

The filtrate was loaded into a preconditioned Oasis MCX column (150 mg, Waters Corp., San Francisco, CA, USA). After the column was washed with 1-M formic acid, ABA fractions were eluted with $5-\mathrm{mL}$ methanol. The eluate was evaporated in vacuo and dissolved in distilled water. The solution was adjusted to $\mathrm{pH} 2.5$ with $\mathrm{HCl}$, and the aqueous solution was partitioned three times against ethyl acetate. The pooled ethyl acetate phase was dried over anhydrous $\mathrm{Na}_{2} \mathrm{SO}_{4}$. The dried ethyl acetate phase was then evaporated in vacuo and dissolved in methanol. Each measurement had two to three replicates.

Methylated ABA was quantified using GC-MS-SIM (QP5000; Shimadzu) equipped with a fused-silica capillary column $(0.25 \mathrm{~mm}$ i.d. $\times 30 \mathrm{~m}$, ZB-1; Phenomenex Inc., Torrance, CA, USA). Selected ion monitoring was conducted at $m / z$ 190/194 for $\mathrm{ABA} /\left[{ }^{2} \mathrm{H}_{6}\right] \mathrm{ABA}$.

Experiment 2: Effects of ethylene synthesis inhibitor treatment on berries before the onset of maturation on the maturation, ethylene evolution, and ABA concentration

Four 27-year-old northern highbush blueberry 'Jersey' bushes growing at Takizawa Farm at the Field
Science Center of Iwate University, Japan, were used in 2011. Two canes were selected per bush, and one cane was used for each treatment per bush. Approximately 160 berries at the MG stage were selected per treatment and tagged just before treatment. The berries were sprayed with aminoethoxyvinylglycine (AVG) (OlChemIm.), which is an ethylene synthesis inhibitor, $150 \mathrm{mg} \cdot \mathrm{L}^{-1}$ containing $0.1 \%$ Tween 20 on 15 July. Untreated berries were used as the control.

The berries were collected randomly on $0,4,7$, and 10 DAT to determine the quality, ethylene evolution, and $\mathrm{ABA}$ concentration. These were measured in the same way as in Experiment 1, except that 2-3 berries were used per replication for evaluation of the berry quality, and 4 berries per replication for measuring ethylene evolution and the ABA concentration in triplicate.

Experiment 3: Effects of ABA synthesis inhibitor treatment on berries before the onset of maturation on the maturation, ethylene evolution, and ABA concentration

Three 28-year-old northern highbush blueberry 'Jersey' bushes growing at Takizawa Farm at the Field Science Center of Iwate University, Japan, were used in 2012. Two canes were selected per bush, and one cane was used for each treatment per bush. Approximately 160 berries at the MG stage were selected per treatment and tagged just before treatment. The berries were sprayed with nordihydroguaiaretic acid (NDGA) (MP Biomedicals, LLC., France), which is an ABA synthesis inhibitor, $300 \mathrm{mg} \cdot \mathrm{L}^{-1}$ containing $0.1 \%$ Tween 20 on 11 July. Untreated berries were used as the control. The berries were randomly collected on $0,4,712$, and 16 DAT to determine the quality, ethylene evolution, and ABA concentration. These were measured in the same way as in Experiment 1, except that 2-3 berries per replication were used for measuring ethylene evolution.

\section{Statistical analysis}

Statistical analyses were performed using JMP 7 (SAS institute, Cary, NC, USA). Data were analyzed by Tukey-Kramer HSD test or $t$-test at the 5\% level of significance.

\section{Results}

Experiment 1: Effects of ethephon and ABA treatment on berries before the onset of maturation on the maturation, ethylene evolution, and ABA concentration

Berries were harvested at 16 DAT in the control and ABA treatment, and 7 DAT in the ethephon treatment (Fig. 1A). The berry weight, soluble solid content, and juice $\mathrm{pH}$ in the control and ABA treatment increased at 16 DAT and those in the ethephon treatment increased at 7 DAT (Fig. 1A, C, D). Berry firmness decreased in the control and ABA treatment at 16 DAT, and in the ethephon treatment at 7 DAT (Fig. 1B).

Ethylene evolution increased slightly in the control and $\mathrm{ABA}$ treatment at 16 and $11 \mathrm{DAT}$, respectively, and 

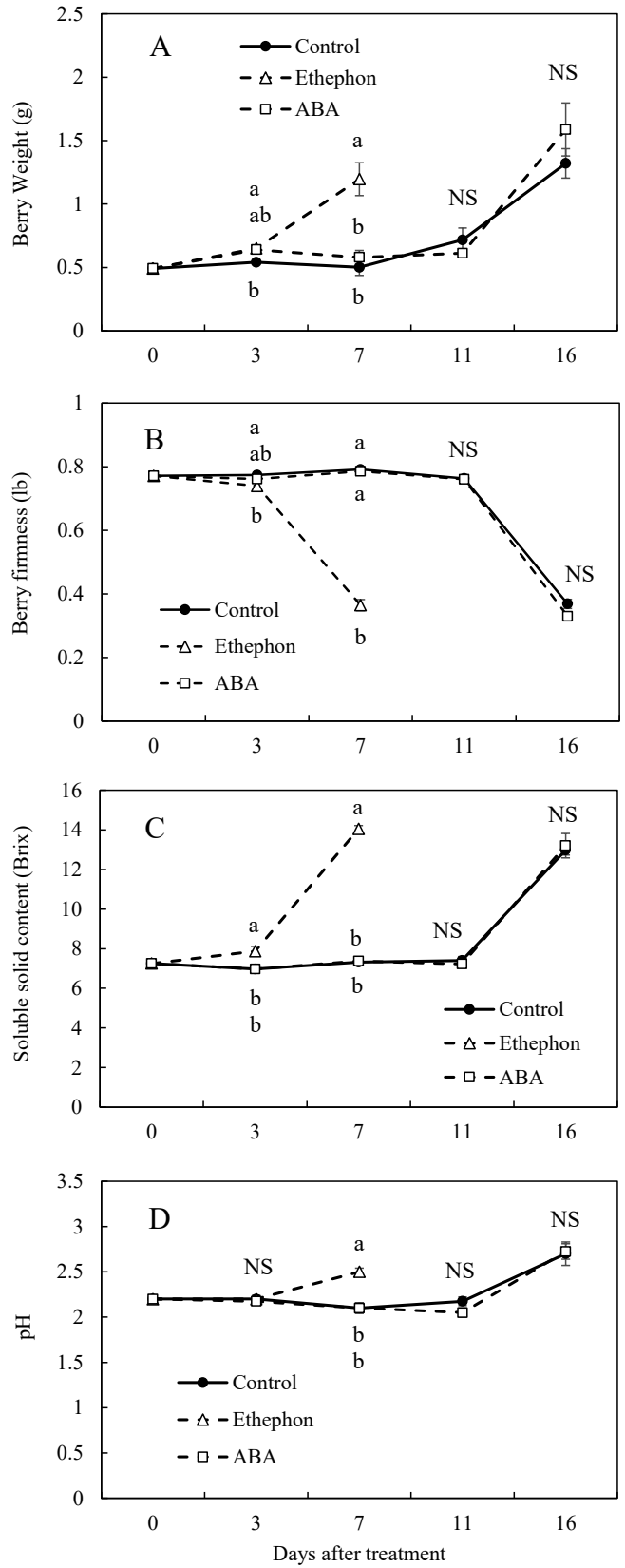

Fig. 1. Effect of ethephon and abscisic acid (ABA) on berries before the onset of maturation: on berry weight (A), firmness (B), soluble solid content (C), and $\mathrm{pH}(\mathrm{D})$ in 'Jersey'. Data of berry weight $(n=2-3)$, firmness $(n=3-4)$, soluble solid content, and $\mathrm{pH}(\mathrm{n}=4)$ show the mean \pm standard error $(\mathrm{SE})$. Different letters and NS indicate significant differences at $P<0.05$ and non-significance by Tukey-Kramer HSD test or $t$-test, respectively.

increased rapidly in the ethephon treatment at 3 DAT (Fig. 2A). The peak ethylene evolution was three times higher in the ethephon treatment than in the control and ABA treatment. The ABA concentration in the control increased from 7 to 11 DAT (Fig. 2B). In berries treated with ethephon or the ABA treatment, the ABA concentration increased at 3 DAT. The ABA concentration in the ethephon treatment subsequently increased until 7
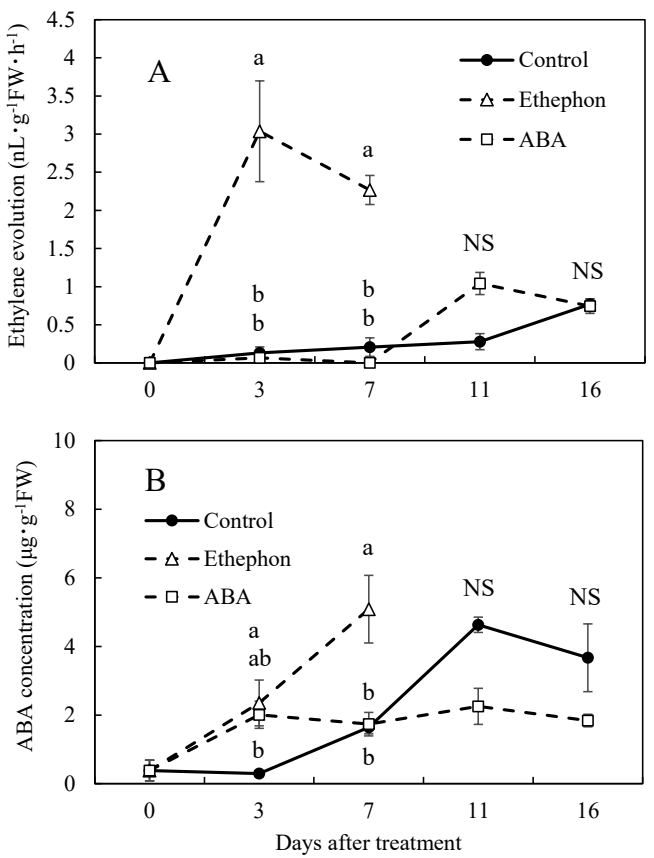

Fig. 2. Effect of ethephon and ABA on berries before the onset of maturation: on ethylene evolution (A) and $\mathrm{ABA}$ concentration (B) in 'Jersey'. Data of ethylene evolution $(n=4)$ and ABA concentration $(n=2-3)$ show the mean \pm SE. Different letters and NS indicate significant differences at $P<0.05$ and nonsignificance by Tukey-Kramer HSD test or $t$-test, respectively.

DAT, while that in the ABA treatment was constant until 16 DAT. The peak ABA concentrations were 2.5 times higher in the ethephon treatment and control than in the $\mathrm{ABA}$ treatment.

Experiment 2: Effects of ethylene synthesis inhibitor treatment on berries before the onset of maturation on the maturation, ethylene evolution, and ABA concentration

The berries were harvested at 7 and 10 DAT in the control and AVG treatment, respectively (Fig. 3A). The berry weight and soluble solid content in the control and AVG treatment were increased at 7 and 10 DAT, respectively (Fig. 3A, C). The berry firmness decreased at 7 DAT in the control and at 10 DAT in the AVG treatment (Fig. 3B). The juice $\mathrm{pH}$ in the control increased at 7 DAT, while that in the AVG treatment remained constant during the investigation period (Fig. 3D).

Ethylene evolution increased rapidly in the control and slowly in the AVG treatment at 7 DAT (Fig. 4A). The peak level of ethylene evolution was half that of the control in the AVG treatment. The ABA concentration in the control and AVG treatment increased at 7 and 10 DAT, respectively (Fig. 4B). There was no difference in the peak ABA concentration between the control and AVG treatment. 

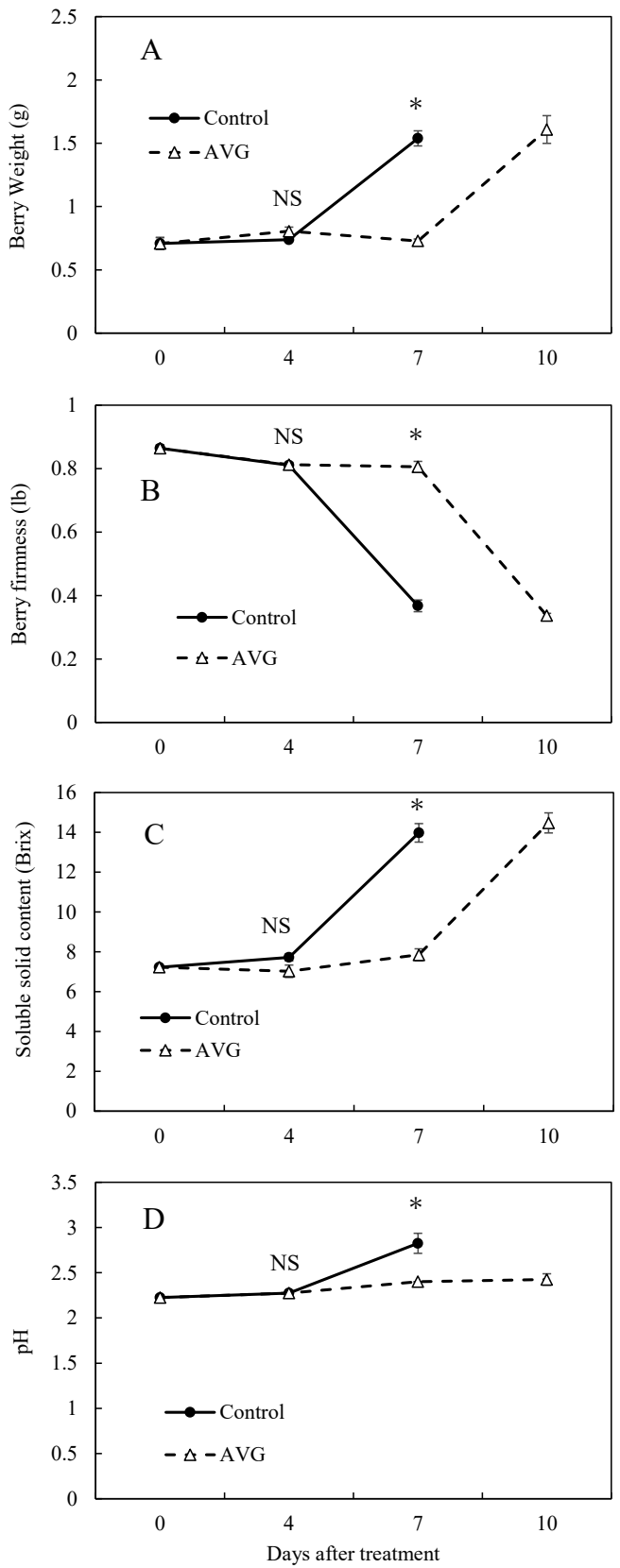

Fig. 3. Effect of aminoethoxyvinylglycine (AVG) on berries before the onset of maturation: on berry weight (A), firmness (B), soluble solid content (C), and $\mathrm{pH}(\mathrm{D})$ in 'Jersey'. Data of berry weight $(n=3)$, firmness, soluble solid content and $\mathrm{pH}(\mathrm{n}=4)$ show the mean \pm SE. * and NS indicate significant differences at the $P<0.05$ level and non-significance by $t$-test, respectively.

Experiment 3: Effects of ABA synthesis inhibitor treatment on berries before the onset of maturation on the maturation, ethylene evolution, and ABA concentration

The NDGA treatment showed no effect on the harvest time or berry weight (Fig. 5A). There were significant differences in the berry firmness, soluble solid content, and juice $\mathrm{pH}$ (Fig. 5B, C, D), but the differences were small. Ethylene evolution in the NDGA treatment did not differ from the control (Fig. 6A). The
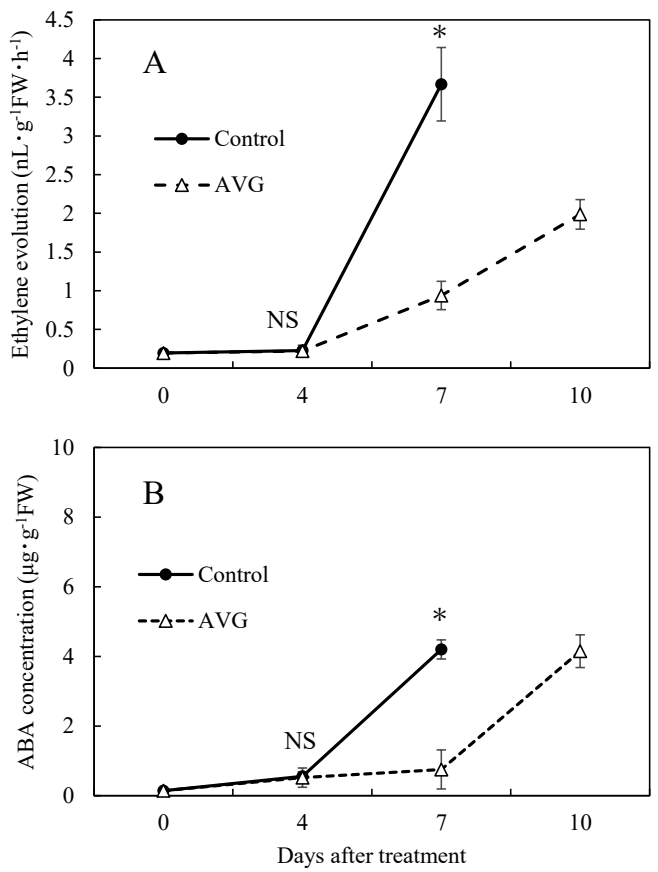

Fig. 4. Effect of AVG on berries before the onset of maturation: on ethylene evolution (A) and ABA concentration (B) in 'Jersey'. Data of ethylene evolution $(n=4)$ and ABA concentration $(\mathrm{n}=3)$ show the mean $\pm \mathrm{SE} .{ }^{*}$ and NS indicate significant differences at $P<0.05$ and non-significance by $t$-test, respectively.

ABA concentration in the control and NDGA treatment increased from 7 DAT and peaked at 12 DAT (Fig. 6B). However, the ABA concentration was lower in the NDGA treatment than in the control at 7 and 16 DAT.

\section{Discussion}

Eck (1970) reported that ethrel at $240 \mathrm{ppm}$ on 'Weymouth' and 1,920 ppm on 'Blueray' highbush blueberry applied as a foliar spray to blueberry two weeks before the first harvest resulted in a significant increase in the percentage of first harvest to the total yield. Ban et al. (2007) indicated that the application of $200 \mathrm{ppm}$ of ethephon to clusters at the onset of the first berry coloration promoted maturation in 'Tifblue' rabbiteye blueberry. Warren et al. (1973) showed that dipping clusters into $2,000-8,000 \mathrm{ppm}$ of ethephon at the onset of stage III advanced berry maturity in 'Morrow' highbush blueberry. Thus, there is no doubt that ethylene application promotes the maturation of blueberry.

In the present study, the effects of ethephon on the maturation of berries were consistent with previous reports. In addition, the ethephon treatment increased not only ethylene evolution but also the ABA concentration in berries.

There are few reports on the effect of ethylene on the ABA concentration in climacteric fruit. Zhang et al. (2009a) demonstrated that $100-\mu \mathrm{M}$ 1aminocyclopropane-1-carboxylic acid (ACC) treatment 

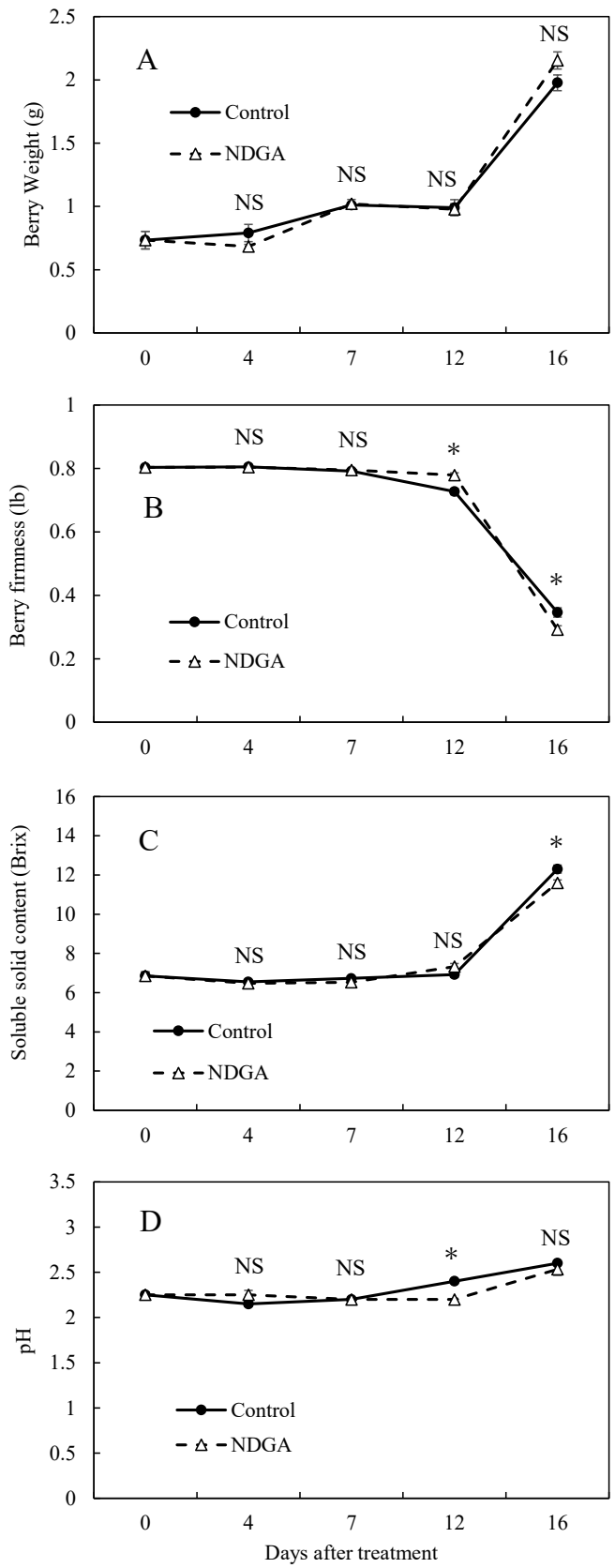

Fig. 5. Effect of nordihydroguaiaretic acid (NDGA) on berries before the onset of maturation: on berry weight (A), firmness (B), soluble solid content (C), and pH (D) in 'Jersey'. Data of berry weight $(n=2-3)$, firmness $(n=2-4)$, soluble solid content $(\mathrm{n}=4)$ and $\mathrm{pH}(\mathrm{n}=3)$ show the mean \pm SE. * and NS indicate significant differences at $P<0.05$ and non-significance by $t$-test, respectively.

increased ethylene evolution but did not increase the ABA concentration in the MG stage of tomato. Lara and Vendrell (2000b) examined the effect of exposure to ethylene on 'Granny Smith' apple fruits collected at three different maturity stages. The ethylene treatment increased the ABA concentration in pulp at 1 month before commercial harvest, and inhibited or did not increase ABA accumulation at 2 months before commercial harvest and at harvest.
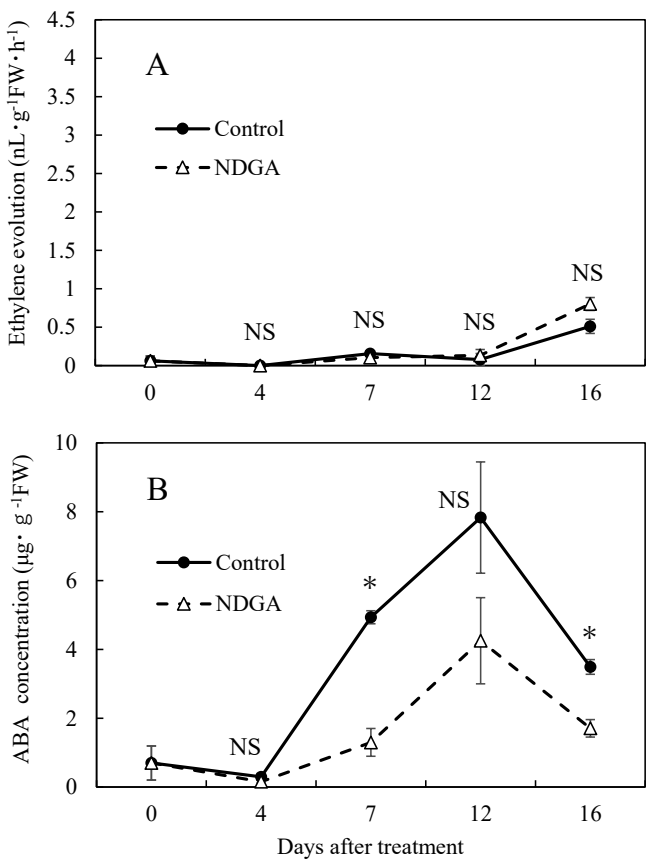

Fig. 6. Effect of nordihydroguaiaretic acid (NDGA) on berries before the onset of maturation: on ethylene evolution (A) and ABA concentration (B) in 'Jersey'. Data of ethylene evolution $(\mathrm{n}=4)$ and ABA concentration $(\mathrm{n}=2-3)$ show the mean \pm SE. * and NS indicate significant differences at $P<0.05$ and nonsignificance by $t$-test, respectively.

In many apple cultivars, the fruits at two months before commercial harvest are at an immature stage in which ethylene evolution stays at a very low level, and the fruit at 1 month before commercial harvest is when ethylene evolution begins to increase (Kondo et al., 1991; Suzuki et al., 1997c). The peak of ethylene evolution was not detected in the control in the present study, since the collecting interval was 4-5 days during the investigation period. However, it could be estimated that ethylene evolution peaked after 11 DAT according to the change in berry firmness and soluble solid content in the control, and average temperature in 2010. The maturing stage of 11 DAT and 16 DAT were estimated as the green-pink (GP) and ripe (R) stage, respectively, according to the change in ethylene evolution, berry firmness, and soluble solid content (Goto et al., 2013). The peak of ethylene evolution occurred in the bluepink and blue stages, which are between the pink-green and R stage (Goto et al., 2013). Suzuki et al. (1997a) indicated that the GP stage is 6 days before harvest. The days from the GP to R stage, however, may have been much shorter in 2010, because the average temperature was $2.4^{\circ} \mathrm{C}$ higher in 2010 than in an average year from 14 July (0 DAT) to 30 July (16 DAT). Although the ethephon treatment progressed the time of increased ABA concentration in berries, the effect of ethylene on the ABA concentration may have differed depending on the treatment time.

Although, there are a few reports in the literature 
about the effect of an ethylene synthesis inhibitor on blueberry maturation, the effects of an ethylene synthesis inhibitor on the ABA concentration of berries has not yet been reported. Dekazos (1979, cited by Eck, 1988) found that ripening was delayed about a week by AVG treatment in rabbiteye blueberry. Suzuki et al. (1997b) reported aminooxyacetic acid treatment on berries about 10 days before the onset of maturation did not inhibit ethylene evolution but delayed maturation in 'Jersey'. In the present study, AVG treatment on berries before the onset of maturation delayed maturation and delayed the time of the increase not only of ethylene evolution but also of the ABA concentration. Mou et al. (2016) reported that the increase in the ABA concentration was delayed in tomato fruits treated with 1-MCP immediately after ABA treatment at the MG stage, indicating ethylene might be essential for the induction of ABA synthesis. Zhang et al. (2009a) determined that the ABA concentration increased similarly in both the $\mathrm{ABA}$ treatment alone and together with 1-MCP when tomato was treated with 1-MCP 4 days after $\mathrm{ABA}$ treatment at the MG stage. Thus, it was suggested that the inhibition of ethylene synthesis before the onset of maturation, when the ABA concentration has not yet increased, inhibits ABA synthesis resulting in a delay in maturation. The results of the ethephon and AVG treatment before the onset of maturation indicate that ethylene initiates maturation and is essential to ABA synthesis at the MG stage before the onset of maturation in blueberry.

The ABA concentration rapidly increases at maturation in blueberry (Goto et al., 2013; Kobashi et al., 2002; Zifkin et al., 2012), suggesting that both ABA and ethylene might be involved in the maturation of blueberry. Also, ABA treatment of apple fruit at the pre-climacteric stage promoted maturation (Vendrell and Buesa, 1989). In mango, ABA treatment during ripening progressed coloration and softening of the pericarp (Zaharah et al., 2012), and the maturation of banana was promoted by ABA treatment (Jiang et al., 2000). These reports show that ABA accelerates the progress of maturation in climacteric fruit, including blueberry. Oh et al. (2018) reported that fruit clusters dipped into $1 \mathrm{~g} \cdot \mathrm{L}^{-1}$ ABA solution six weeks after full bloom showed stimulated berry coloration and softening. In contrast, Buran et al. (2012) revealed that the application of 200 or $400 \mathrm{ppm}$ of ABA to the same cluster three times delayed maturation in southern highbush blueberries 'Star' and 'Windsor'. It was, however, difficult to judge the precise effects of ABA on the maturation of blueberry, since ABA was applied repeatedly to berries at different developmental stages.

In the present study, berries treated with $\mathrm{ABA}$ at the same developmental stage before the onset of maturation did not show maturation promotion. Thus, the effect of ABA on the maturation of blueberry differs among reports. It is suggested that the effect of $\mathrm{ABA}$ on maturation differs depending on the developmental stage, and that the period during which ABA can promote maturation is shorter than that by ethylene in blueberry. Although, in this study, berries were treated with $30 \mathrm{mg} \cdot \mathrm{L}^{-1} \mathrm{ABA}$, which is almost the same concentration as Zhang et al. (2009b), the ABA concentration was lower compared with the other reports applying $\mathrm{ABA}$ to blueberry. Therefore, the ABA concentration might be too low to promote the maturation of blueberry in the present study. Further investigation is necessary to elucidate the effects of $\mathrm{ABA}$ at higher concentrations on the maturation of blueberry.

ABA treatment temporarily increased the ABA level in berries after six weeks of full bloom, and stimulated the process of maturation in 'Jersey' (Oh et al., 2018). There are no reports on the effect of ABA treatment on ethylene evolution in blueberry. In the present study, ABA treatment slightly increased the ABA concentration at 3 DAT, and increased the ethylene evolution at 11 DAT in berries. The increase in the ABA concentration may be a result of measuring the applied ABA itself, not promoting $\mathrm{ABA}$ synthesis by $\mathrm{ABA}$ treatment. In any case, it is considered that increased ethylene evolution may occur naturally, not by $\mathrm{ABA}$ treatment, since the time of the increase in ethylene evolution was 7 days later than that of the increase in the ABA concentration. In immature peach, characterized by system I ethylene production and low levels of ABA, it is plausible that exogenous ABA has a different effect than at a more advanced developmental stage, when the ethylene biosynthetic apparatus is more advanced and ABA biosynthesis is also increasing (Bonghi et al., 2011). In peach, ABA treatment inhibited the ethylene biosynthetic machinery and the progression of maturation in fruit at 102 days after full bloom (DAFB), producing no detectable ethylene; the opposite effects were observed in fruit at 115 DAFB producing no detectable ethylene and at 118 DAFB producing ethylene (Soto et al., 2013).

Similarly, it was considered that ABA did not increase ethylene evolution with ABA treatment of berries at the developmental stage before the increase of ethylene evolution in this study. In ABA-treated fruit, 9-cis-epoxycarotenoid dioxygenase, which is the main rate-limiting step in ABA biosynthesis, may be either down-regulated at the pre-climacteric stage or upregulated at the climacteric stage (Soto et al., 2013). It is considered that the ABA concentration did not rapidly increase in treated $\mathrm{ABA}$ on fruits before the onset of maturation in blueberry, which is classified as a climacteric fruit showing a double sigmoid growth curve similar to peach in this study. It was suggested that as ethylene evolution and the ABA concentration increased, maturation was promoted if berries were treated with $\mathrm{ABA}$ at the time of increasing ethylene evolution and ABA concentration. However, it is difficult to judge the effect of ABA on promoting matura- 
tion, since the ABA concentration in blueberry reached a peak within 10 days from the beginning of the ABA increase, and the maturation rapidly progressed.

NDGA treatment of harvested mango inhibited ethylene biosynthesis and the progress of maturation (Zaharah et al., 2013). Fluridone and NDGA treatment of tomato fruit at MG inhibited the ABA concentration and ethylene evolution and delayed maturation (Zhang et al., 2009a). The application of NDGA to tomato at MG significantly blocked the accumulation of ABA and kept the level consistently low for a very long time, which may ultimately lead to a delayed triggering of ethylene synthesis (Mou et al., 2016). Thus, many studies have shown that ABA may control ethylene biosynthesis in fruit maturation. The NDGA treatment delayed the increase in the $\mathrm{ABA}$ concentration and decreased the peak value in this study, while it did not affect ethylene evolution and maturation. Application of $0.5 \mathrm{~mL}$ of $100 \mu \mathrm{M}$ NDGA in Zhang et al. (2009a) and $25 \mu \mathrm{L}$ of $1 \mathrm{mM}$ NDGA in Mou et al. (2016) into the fruit with a syringe produced a low ABA concentration, which was maintained for 12 and 18 days, respectively.

Although NDGA at a concentration of about 3 and $1 / 3$ times the above reports were sprayed on berries attached on the bush in the present study, the increase in the ABA concentration was not sufficiently inhibited. In the future, it will be necessary to improve the NDGA treatment method, such as by repeated spraying. Jiang et al. (2000) found that the initiation of ripening in climacteric fruit is triggered by ethylene, while ABA may act as a coordinator during the climacteric process. Meanwhile, Zhang et al. (2009a) reported that ABA triggers ethylene production in tomato fruit ripening, and ethylene is more important for the later ripening stage. In the present study, AVG and NDGA temporarily inhibited ethylene evolution and the ABA concentration, respectively. Although the temporary inhibition of ethylene evolution led to delayed onset and progress of maturation, the temporary inhibition of the ABA concentration did not affect the onset and progress of maturation. Thus, we did not observe any effect of ABA on the onset and progress of maturation in blueberry. These results suggest that ethylene may be more important than $\mathrm{ABA}$ for the onset and progress of maturation in blueberry.

Maturation progressed more rapidly in blueberry showing a double-sigmoid growth curve than in fruit showing a single-sigmoid growth curve. Moreover, the period from flowering to harvesting in blueberry is 60 80 days, shorter than that in peach and grape among fruit trees showing a double-sigmoid growth curve. The changes in ethylene and ABA may not be properly understood, since the progress of maturation is very rapid in blueberry, and the berry-collecting interval was 3-5 days in the present study. The time of increased ABA concentration is earlier than that of ethylene evolution in tomato (Martinez-Madrid et al., 1996) and peach
(Zhang et al., 2009b). The ABA concentration peaked 3 weeks earlier in tomato (Martinez-Madrid et al., 1996), and 30 days before the ethylene evolution peaked in peach (Zhang et al., 2009b). On the other hand, the change in the ABA concentration in the pulp apparently showed a similar pattern to increased ethylene evolution in an early-harvest apple cultivar (Kondo et al., 1991). The time of the increase and peak in the ABA concentration resembled that of ethylene in blueberry (Goto et al., 2013). Thus, climacteric fruit may be classified into two types for the relation between the peak time of ABA concentration and ethylene evolution. The interaction between ethylene and ABA may differ in each type.

In apple, the change in the ACC concentration and ABA concentration differed in pulp and peel at the maturation stage (Kondo et al., 1991; Lara and Vendrell, 2000a). In the present study, ethylene evolution and the ABA concentration of whole fruit were determined. In the future, the relationship between ethylene and $\mathrm{ABA}$ in maturation may be clarified in more detail, determining the ethylene evolution and $\mathrm{ABA}$ concentration of pulp and peel in blueberry.

In conclusion, our results suggest that ethylene, not $\mathrm{ABA}$, induced maturation of blueberry at the MG stage, and that ethylene is more closely involved than ABA in the progress of the whole maturation process.

\section{Literature Cited}

Ban, T., M. Kugishima, T. Ogata, S. Shiozaki, S. Horiuchi and H. Ueda. 2007. Effect of ethephon (2-chloroethylphosphonic acid) on the fruit ripening characters of rabbiteye blueberry. Sci. Hortic. 112: 278-281.

Bonghi, C., L. Trainotti, A. Botton, A. Tadiello, A. Rasori, F. Ziliotto, V. Zaffalon, G. Casadoro and A. Ramina. 2011. A microarray approach to identify genes involved in seedpericarp cross-talk and development in peach. BMC Plant Biol. 11: 107. DOI: 10.1186/1471-2229-11-107.

Buran, T. J., A. K. Sandhu, A. M. Azeredo, A. H. Bent, J. G. Williamson and L. Gu. 2012. Effects of exogenous abscisic acid on fruit quality, antioxidant capacities, and phytochemical contents of southern high bush blueberries. Food Chem. 132: $1375-1381$.

Chung, S. W., D. K. Yu, H. D. Oh, J. H. Ahn, J. H. Huh and H. J. Lee. 2019. Transcriptional regulation of abscisic acid biosynthesis and signal transduction, and anthocyanin biosynthesis in 'Bluecrop' highbush blueberry fruit during ripening. PLoS ONE 14: e0220015. DOI: 10.1371/journal. pone. 0220015 .

Coombe, B. G. and C. R. Hale. 1973. The hormone content of ripening grape berries and the effects of growth substance treatments. Plant Physiol. 51: 629-634.

Dekazos, E. D. 1979. Storage studies and quality evaluation of explosion puffed rabbiteye blueberries. p. 271-288. In: J. N. Moore (ed.). Proc. 4th Amer. Blueberry Res. Workers Conf. Univ. of Arkansas.

Eck, P. 1970. Influence of ethrel upon highbush blueberry fruit ripening. HortScience 5: 23-25.

Eck, P. 1988. Blueberry science. p. 89. Rutgers Univ. Press, New Brunswick and London.

Frenkel, C. 1972. Involvement of peroxidase and indole-3-acetic 
acid oxidase isozymes from pear, tomato, and blueberry fruit in ripening. Plant Physiol. 49: 757-763.

Goto, R., M. Watanabe, M. Murakami, S. Sagawa, S. Komori and A. Suzuki. 2013. The changes in IAA and ABA concentration and ethylene evolution during fruit development in blueberry. Hort. Res. (Japan) 12: 165-171 (In Japanese with English abstract).

Hall, I. V. and F. R. Forsyth. 1967. Respiration rates of developing fruits of the lowbush blueberry. Can. J. Plant Sci. 47: 157-159.

Hiwasa-Tanase, K. and H. Ezura. 2014. Part1. Physiology and metabolism. Fruit ripening. p. 1-14. In: P. Nath, M. Bouzayen, A. K. Mattoo and J. C. Pech (eds.). Fruit Ripening: physiology, signalling and genomics. $\mathrm{CAB}$ International publishing, USA.

Ismail, A. A. and W. J. Kender. 1969. Evidence of a respiratory climacteric in highbush and lowbush blueberry fruit. HortScience 4: 342-344.

Jiang, Y., D. C. Joyce and A. J. Macnish. 2000. Effect of abscisic acid on banana fruit ripening in relation to the role of ethylene. J. Plant Growth Regul. 19: 106-111.

Kobashi, K., S. Sugaya, M. Fukushima and S. Iwahori. 2002. Sugar accumulation in highbush blueberry fruit as affected by artificial pollination with different pollen sources in relation to seed number, invertase activities and ABA content. Acta Hortic. 574: 47-51.

Kobashi, K., S. Sugaya, H. Gemma and S. Iwahori. 2001. Effect of abscisic acid (ABA) on sugar accumulation in the flesh tissue of peach fruit at the start of the maturation stage. Plant Growth Regul. 35: 215-233.

Kobayashi, M., N. Horiuchi, M. Omura, J. Che and I. Ogiwara. 2011. Comparison of fruit quality between individual and cluster harvesting in four blueberry cultivars. Hort. Res. (Japan) 10: 507-512 (In Japanese with English abstract).

Kondo, S., J. Uthaibutra and H. Gemma. 1991. Comparison of 1-aminocyclopropane-1-carboxylic acid, abscisic acid and anthocyanin content of some apple cultivars during fruit growth and maturation. J. Japan. Soc. Hort. Sci. 60: 505511.

Lara, I. and M. Vendrell. 2000a. Changes in abscisic acid levels, ethylene biosynthesis, and protein patterns during fruit maturation of 'Granny Smith' apples. J. Amer. Soc. Hort. Sci. 125: $183-189$.

Lara, I. and M. Vendrell. 2000b. Development of ethylenesynthesizing capacity in preclimacteric apples: Interaction between abscisic acid and ethylene. J. Amer. Soc. Hort. Sci. 125: 505-512.

Lin, S. and C. S. Walsh. 2008. Studies of the "tree factor" and its role in the maturation and ripening of 'Gala' and 'Fuji' apples. Postharvest Biol. Technol. 48: 99-106.

Lipe, J. A. 1978. Ethylene in fruits of blackberry and rabbiteye blueberry. J. Amer. Soc. Hort. Sci. 103: 76-77.

Martinez-Madrid, M. C., M. Serrano, F. Riquelme and F. Romojaro. 1996. Polyamines, abscisic acid and ethylene production in tomato fruit. Phytochemistry 43: 323-326.

Miyashita, C., Y. Koito and I. Ogiwara. 2019. Utility of Parthenocarpic interspecific hybrids between Vaccinium corymbosum and Vaccinium virgatum for breeding blueberry cultivars suitable for cluster harvesting. Hort. J. 88: 180-188.

Mou, W., D. Li, J. Bu, Y. Jiang, Z. U. Khan, Z. Luo, L. Mao and T. Ying. 2016. Comprehensive analysis of ABA effects on ethylene biosynthesis and signaling during tomato fruit ripening. PLoS ONE 11: e0154072. DOI: 10.1371/journal. pone. 0154072 .
Oh, H. D., D. J. Yu, S. W. Chung, S. Chea and H. J. Lee. 2018. Abscisic acid stimulates anthocyanin accumulation in 'Jersey' highbush blueberry fruits during ripening. Food Chem. 244: 403-407.

Rogiers, S. Y. and N. R. Knowles. 1999. A comparison of preharvest and postharvest ethylene production and respiration rates of saskatoon (Amelanchier alnifolia nutt) fruit during development. Can. J. Bot. 77: 323-332.

Shimura, I., M. Kobayashi and S. Ishikawa. 1986. Characteristics of fruit growth and development in highbush and rabbiteye blueberries (Vaccinium corymbosum L. and V. ashei Reade) and the differences among their cultivars. J. Japan. Soc. Hort. Sci. 55: 46-50.

Soto, A., K. B. Ruiz, D. Ravaglia, G. Costa and P. Torrigiani. 2013. ABA may promote or delay peach fruit ripening through modulation of ripening- and hormone-related gene expression depending on the developmental stage. Plant Physiol. Biochem. 64: 11-24.

Suzuki, A., T. Kikuchi and K. Aoba. 1997a. Changes of ethylene evolution, ACC content, ethylene forming enzyme activity and respiration in fruits highbush blueberry. J. Japan. Soc. Hort. Sci. 66: 23-27.

Suzuki, A., T. Kikuchi and K. Aoba. 1997b. Effects of ethylene on fruit set and maturation of highbush blueberry (Vaccinium corymbosum L.). J. Japan. Soc. Hort. Sci. 66: 267-727.

Suzuki, A., A. Takahashi, K. Aoba, T. Masuda and Y. Kashimura. 1997c. Comparison in ethylene biosynthesis among attached and detached fruits of several apple cultivars during maturation. J. Japan. Soc. Hort. Sci. 66: 495-503.

Vendrell, M. and C. Buesa. 1989. Relationship between abscisic acid content and ripening of apples. Acta Hortic. 258: 389396.

Walsh, C. S. 1977. The relationship between endogenous ethylene and abscission of mature apple fruits. J. Amer. Soc. Hort. Sci. 102: 615-619.

Warren, J. M., W. E. Ballinger and C. M. Mainland. 1973. Effects of ethephon upon fruit development and ripening of highbush blueberries in the greenhouse. HortScience 8: 504-507.

Windus, N. D., V. G. Shutak and R. E. Gough. 1976. $\mathrm{CO}_{2}$ and $\mathrm{C}_{2} \mathrm{H}_{4}$ evolution by highbush blueberry. HortScience 11: 515 517.

Zaharah, S. S., Z. Singh, G. M. Symons and J. B. Reid. 2012. Role of brassinosteroids, ethylene, abscisic acid, and indole-3-acetic acid in mango fruit ripening. J. Plant Growth Regul. 31: 363-372.

Zaharah, S. S., Z. Singh, G. M. Symons and J. B. Reid. 2013. Mode of action of abscisic acid in triggering ethylene biosynthesis and softening during ripening in mango fruit. Postharvest Biol. Technol. 75: 37-44.

Zhang, M., P. Leng, G. Zhang and X. Li. 2009a. Cloning and functional analysis of 9-cis-epoxycarotenoid dioxygenase (NCED) genes encoding a key enzyme during abscisic acid biosynthesis from peach and grape fruits. J. Plant Physiol. 166: 1241-1252.

Zhang, M., B. Yuan and P. Leng. 2009b. The role of ABA in triggering ethylene biosynthesis and ripening of tomato fruit. $\mathrm{J}$. Exp. Bot. 60: 1579-1588.

Zifkin, M., A. Jin, J. A. Ozga, L. I. Zaharia, J. P. Schernthaner, A. Gsell, S. R. Abrams, J. A. Kennedy and C. P. Constabel. 2012. Gene expression and metabolite profiling of developing highbush blueberry (Vaccinium corymbosum L.) fruit indicates transcriptional regulation of flavonoid metabolism and activation of abscisic acid metabolism. Plant Physiol. 158: 200-224. 